\title{
PARTIAL PURIFICATION AND CHARACTERIZATION BY FLUORIMETRY AND SPECTROPHOTOMETRY OF BUFFALO LACTOFERRIN
}

\author{
Edson Ferreira da Silva*, Milena Bandeira de Melo, Marta Ramos Lima, José Leandro da \\ Silva Duarte, Carmem Lucia de Paiva e Silva Zanta, Sonia Salgueiro Machado, Fabiane \\ Caxico de Abreu Galdino. \\ edsonfesilva1@gmail.com \\ UNIVERSIDADE FEDERAL DE ALAGOAS - UFAL
}

Introduction: Lactoferrin (Lf) is a iron ligating glycoprotein with a molecular mass of approximately $80 \mathrm{kDa}$, present in mammalian whey, belonging to the transferrin family. Lf can be found in various mucous secretions, such as tears, saliva, gastrointestinal fluids, urine and seminal fluid, as well as in secondary neutrophil granules, being released in places where there is an inflammatory response. Lf is a multifunctional protein possessing functions such as antibacterial, antiviral, antifungal, anti-inflammatory and immunomodulatory activities. This study aimed to purify, characterizing buffalo milk lactoferrin by monitoring the purification by spectroscopic techniques as well as investigating the interaction of protein with antibiotic amoxicillin. Materials and methods: The processing of the buffalo milk began with the separation of fat by centrifugation. The skimmed milk was acidified with $\mathrm{HCl} 0.1 \mathrm{M}$ up to $\mathrm{pH} 4.6$, obtaining acidified whey and the sour serum was neutralized with $\mathrm{NaOH} 0.1 \mathrm{M}$ until pH 6.8 and then centrifuged. The supernatant was submitted to saline precipitation profiles of 0 $20 \%, 20-40 \%, 40-60 \%$ and $60-80 \%$ saturation of $\left(\mathrm{NH}_{4}\right)_{2} \mathrm{SO}_{4}$. Fluorimetric analyses of salt fractions were performed under excitation length conditions at $290 \mathrm{~nm}$ and emission wavelengths between 300-550 nm. Spectrophotometric studies were carried out with additions of $100 \mu \mathrm{L}$ of saline fraction $40-60 \%(0.421$ $\mathrm{mg} / \mathrm{mL}), 100 \mu \mathrm{L}$ of purified lactoferrin $(0.421 \mathrm{mg} / \mathrm{mL})$ and $100 \mu \mathrm{L}$ amoxicillin $\left(2.5 \times 10^{-6} \mathrm{~mol}^{\mathrm{L}} \mathrm{L}^{-1}\right)$. Uv-vis absorption spectra were recorded from 190 to $450 \mathrm{~nm}$. Results and discussions: The saline profile of the precipitate was resuspended $40-60 \%$ showed the spectrum of fluorescence extinction characteristic of lactoferrin (peak at $332 \mathrm{~nm}$ ). The $40-60 \%$ precipitate was resuspended and submitted to liquid chromatography in a Sephacryl S-100 gel column. Fractions 12 to 16 showed the fluorescence extinction spectrum characteristic of lactoferrin. SDS-PAGE 8\%, using commercial lactoferrin (SIGMA) as standard, showed the presence of two protein bands in the standard range. The UV-vis spectrum of maximum absorption showed that the interaction between lactoferrin and amoxicillin play an important role, with the decrease and displacement to the red peak on the UV-vis absorption spectrum when compared with that of lactoferrin partially purified with lactoferrin in the presence of the amoxicillin. Conclusion: Buffalo lactoferrin was partially purify by liquid chromatography in a Sephacryl s100 resin and SDS-PAGE 8\%, using commercial lactoferrin (SIGMA) as standard, showed two protein bands in the standard range. Partially purified buffalo lactoferrin exhibited a UV-vis absorption spectrum with two peaks; the first strong peak centered at absorption maximum in the region 220 to $230 \mathrm{~nm}$ is characteristic of the peptide structure and the second peak absorption maximum in the region from 270 to $280 \mathrm{~nm}$ due to conjugate double bonds in tyrosine and tryptophan residues. On assays of partial purified Lf incubated with amoxicillin 
was observed observe a redshift shift from $222.50 \mathrm{~nm}$ to $225 \mathrm{~nm}$ followed by the hypochromic effect on the UV-vis spectrum of maximum absorption of lactoferrin. The UV-visible absorption spectra studies showed that amoxicillin when coupled with lactoferrin induced alterations in the protein structure.

Keywords: Lactoferrin, purification, Fluorimetry, Spectrophotometry. 